\title{
Priming Creativity Through Improvisation on an Adaptive Musical Instrument
}

\author{
Garth Griffin \\ Tufts University \\ 161 College Avenue, Medford, MA 02155 \\ gwgecs.tufts.edu
}

\author{
Robert Jacob \\ Tufts University \\ 161 College Avenue, Medford, MA 02155 \\ jacob@cs.tufts.edu
}

\begin{abstract}
Creativity is a crucial skill in today's knowledge economy, and creativity support tools are a valuable and important area of human-computer interaction research. Passive affective priming has been shown to be an effective means for enhancing creativity. Based on music cognition research, we propose music improvisation as an active and participatory cognitive prime for boosting creative ability. To make music improvisation accessible to all individuals regardless of music expertise, we present a novel instrument with an adaptive interface that facilitates music creation. We demonstrate that improvising music with our adaptive instrument provides an immediate boost to creative ability, even for people without musical training. We quantify the efficacy of interface adaptation techniques for enabling creative expression.
\end{abstract}

\section{Author Keywords \\ Creativity Support Tools; Adaptive Interface; Music \\ Improvisation; Cognitive Priming.}

\section{ACM Classification Keywords}

H.5.1. Information Interfaces and Presentation (e.g. HCI): Multimedia Information Systems; H.5.2. Information Interfaces and Presentation (e.g. HCI): User Interfaces; H.5.5. Information Interfaces and Presentation (e.g. HCI): Sound and Music Computing

\section{General Terms}

Human Factors; Design; Measurement.

\section{INTRODUCTION}

Innovation is the watchword of the knowledge economy. In the technology industry, creativity is recognized as a valuable skill at many levels of the workforce. Engineers are expected to find creative solutions to challenging problems, managers are encouraged to develop creative strategies for getting the best from their employees, and creative company leaders can outplay competitors in a shifting marketplace. A global study from IBM selected creativity as the most important predictor

Permission to make digital or hard copies of all or part of this work for personal or classroom use is granted without fee provided that copies are not made or distributed for profit or commercial advantage and that copies bear this notice and the full citation on the first page. Copyrights for components of this work owned by others than ACM must be honored. Abstracting with credit is permitted. To copy otherwise, or republish, to post on servers or to redistribute to lists, requires prior specific permission and/or a fee. Request permissions from Permissions@acm.org.

C\&C '13, June 17 - 20 2013, Sydney, NSW, Australia

Copyright 2013 ACM 978-1-4503-2150-1/13/06 \$15.00. of success among CEO's [27]. At the same time, creative professionals are increasingly reliant on technological tools for designing, creating, and collaborating. The human-computer interaction (HCI) research community has responded to this need by developing creativity support tools and technologies that enhance creative performance [23].

There have been a number of successful developments in new human-computer interfaces for supporting creativity [23]. One promising new approach for supporting creativity is through cognitive priming. In psychological literature, cognitive priming of positive emotional affect has been shown to increase creative performance $[4,8]$. Lewis et al. introduced this into HCI with affective computational priming, a technique for using digital stimuli to improve later creative performance [14]. This is an example of passive cognitive priming, where subjects were shown a stimulus as a cognitive prime but did not interact with the stimulus directly. Cognitive primes can also take the form of participatory activities, which have the potential to exert greater influence on cognitive state [15].

Substantial psychological research has been done on the effects of music on cognition, but typically researchers look at long-term cognitive benefits from exposure to music, especially in children $[6,7]$. Recent research has shown that healthy adults listening to music experience cognitive benefits in terms of mood and arousal on a shorter timescale [17, 21, 26]. However, these effects do not always manifest immediately and are not necessarily generally applicable. There has been much less investigation of the cognitive impact of music creation, likely because the steep learning curve associated with music performance limits opportunities for behavioral research.

Improvisatory music creation is a good candidate as a participatory cognitive prime for creativity because, in addition to potential benefits in terms of mood and affect arising from the music, the generative nature of improvisation fosters a creative mindset. However, for music improvisation to serve as a cognitive prime for creative professionals, it is necessary that the priming activity be simple, engaging, and accessible without training. This presents a challenge, because playing an instrument typically necessitates years of practice. Building on previous work with adaptive instrument design $[5,16$, 19], we present a novel digital instrument featuring an adaptive interface that facilitates music creation. Our adaptive instrument enables non-musicians and musicians alike to experience the act of music improvisation, which acts as a prime 
to improve performance on a subsequent creative task.

Having developed this adaptive instrument, we face the challenge of quantifying its effectiveness. Often, novel interface designs are evaluated with subjective metrics. By measuring the efficacy of playing the instrument as a cognitive prime, we obtain a quantitative evaluation of the adaptive techniques employed in the design. To measure the impact of the prime on creative ability, we use Guilford's Alternative Uses Task [9]. We chose this task, despite the emergence of newer metrics, because it is one of the most well-established psychological tests of creative ability and was successfully used by Lewis et al. in their recent study of affective priming of creativity [14]. The quantitative assessment complements a traditional subjective evaluation of our instrument.

This enables us to test the following hypotheses:

1. Individuals who are primed by improvising music will subsequently demonstrate higher creative abilities than individuals who are not primed.

2. Individuals who are primed by improvising music using our adaptive interface will subsequently demonstrate higher creative abilities compared to individuals who are primed by improvising music on a non-adaptive interface.

3. Individuals will report greater levels of subjective satisfaction when improvising music on our adaptive interface than when improvising music on a non-adaptive interface.

We find that improvising music does act as a cognitive prime to improve creativity. We find that interface adaptation can increase the improvement, and that the increase varies depending on the type of adaptation. We find that adaptivity can improve levels of satisfaction as well, but this effect is not as strong as the priming. We also report qualitative results for our instrument design, and discuss the potential for large-scale measurement of preferences and trends in musical improvisation for both amateurs and professionals.

Our research makes the following contributions:

1. Quantifying the efficacy of music improvisation as a cognitive prime uncovers possibilities for augmenting current creativity support tools.

2. Quantifying the efficacy of adaptive techniques for empowering creativity supports the development of novel interfaces for creative expression.

3. Our implementation enables us to collect data on the implicit musical preferences of large numbers of participants, paving the way for a more data-driven interface design process.

4. Our work integrates cognitive psychology, music interface design, and creativity support tool design. Furthering this interdisciplinary research enriches the field of HCI by fostering collaboration across scientific disciplines.

In this paper, we outline the background research upon which our work is based, present our adaptive musical instrument, describe our experimental procedure, report the results of our experiments, and conclude with a discussion of future directions and broader implications.

\section{BACKGROUND}

It is difficult to precisely define creativity. Key aspects of creativity include generation of novel ideas, flexibility, and imaginative approaches to problems [2]. The psychological literature on cognitive aspects of creativity is a valuable resource for HCI research in this area. Psychology research suggests that creativity is bolstered by accumulating a variety of ideas beforehand [24]. Drawing on this body of work, Hewett et al. put forward a recommendation for creativity support tools that enable the user to re-formulate the problem in response to changing understanding of the problem state or domain [11]. Lewis et al. successfully used affective computational priming to induce this cognitive variation in subjects, and measured the resulting increases in creative ability [14]. In their study, participants were shown an image that induced negative, neutral or postive affect, and then were assessed for creativite thinking with Guilford's Alternative Uses Task. Lewis et al. found that the digital images served as affective computational primes and could be used in the context of creativity support tools. We extend this idea and propose a new approach where engaging in music improvisation serves as an active and participatory cognitive prime.

There has been much investigation of the links between music, mood, and cognition [6, 7]. In particular, it was demonstrated that listening to music can boost mood and arousal $[17,21,26]$. However, little work has been done to investigate the potential for music creation as a cognitive prime, due to the costly and time-consuming nature of musical training.

With the advent of technologically-aided music creation, this barrier is dissolving. Exploring adaptive instruments and instruments that interact intelligently with the user is an established area of research $[5,16,19]$. Many developments in this area have enabled expert users to express musical ideas that were hitherto out of reach, but this does not necessarily assist individuals who are not music experts. There is also a history of seeking simple, intuitive interfaces that enable non-experts to easily create music $[30,18]$. However, these interfaces often introduce frustrating limits on the range of expressibility. Although intelligent musical instruments have been under investigation for over twenty years [29], improvements in computing power have enabled more sophisticated techniques for computer-aided music creation, which some researchers have begun describing as a new form of musical expression [10]. In this setting, the computer can be thought of as a partner in the creative process. We expand on this to approach the problem of facilitating musical expression across varying levels of musical expertise with a relatively simple physical interface backed by algorithmic techniques that assist the human performer in reaching greater expressive potential. This enables us to include non-musicians in our investigation of music creation as a cognitive prime.

Our quantitative evaluation is based on Guilford's Alternative Uses Task, an established metric of creativity [9]. On this task, subjects are asked to come up with alternative uses for a common household object, such as a brick. Responses are 
Instrument Interface Adapting to Accompanying Music
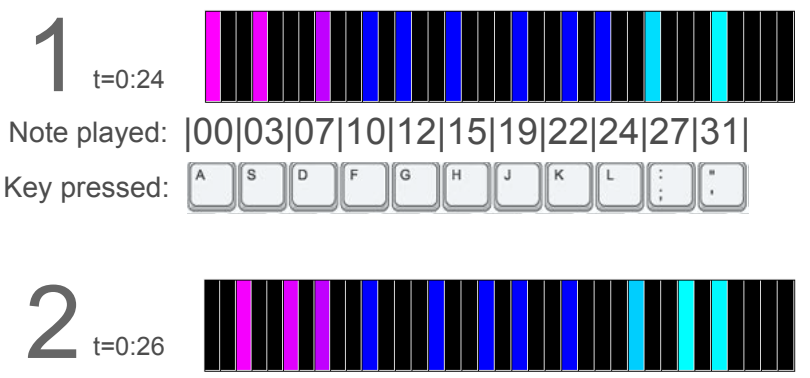

Note played: |02|05|07|10|14|17|19|22|26|29|31|

Key pressed:

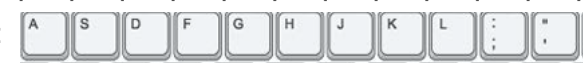

Figure 1. These two screenshots of the web-based instrument show how it adapts to fit with the musical accompaniment. Available notes are highlighted against a backdrop of the chromatic scale. In each screenshot, the home row of keys from a standard computer keyboard are overlaid to show the input-output mapping, with keys labeled with a number representing the pitch at that time. As the underlying harmonic information changes in the musical accompaniment, the instrument adjusts the input-output mapping according to a set of rules derived from principles of music theory.

graded according to subjects' ability to generate many different ideas and according to the originality of subjects' ideas compared to responses from other subjects. This metric has faced some criticism in terms of its ability to provide a complete picture of an individual's creative capacities [25]. In light of this, we treat the metric as a relative measurement of spontaneous idea generation that enables us to quantitatively compare experimental conditions, as opposed to providing nuanced insight into subjects' cognitive processes. While this limits the generality of our results, the metric is still sufficient to show the impact of the techniques we present. This metric was also was successfully used by Lewis et al. to study affective priming of creativity, indicating its feasibility for our work as well [14].

To run our user study, we use Amazon's Mechanical Turk, a web-based micro-task labor market. Mechanical Turk is increasingly popular for scientific work, and past studies have shown that Mechanical Turk is an effective platform for crowd-sourcing user studies [13]. We use Mechanical Turk because it enables much larger sample sizes than are available with conventional participant recruitment procedures. The structure of the Mechanical Turk market makes accurate verification of demographic information impossible. However, previous studies show that Mechanical Turk worker demographics mimic internet users overall $[12,20]$. This provides a reasonable sample set for our research.

\section{MUSIC CREATION INTERFACE}

The goal of our instrument design is to provide a simple interface that retains expressive potential. A trivially simple instrument might consist of a single button marked "go," but no matter how beautiful the music sounded such an instrument would be no more engaging to play than listening to a song on a music player. On the other hand, a sophisticated instrument with many complicated controls might have great expressive power, but would entail a significant learning period, confounding casual use.

To resolve this dilemma, our instrument controls a wide variety of outputs from a relatively small range of inputs. Inputs are given in the form of keypresses from the home row of a standard computer keyboard. When one of these keys is pressed, the instrument plays a note at a particular pitch, similar to how a piano works. Unlike a piano, where each input key corresponds to exactly one output pitch, an input on our instrument corresponds to a family of possible output pitches. The instrument adapts the input-output mapping so that at any given time each input key produces a single output that "sounds good" according to predefined musical rules designed by a music expert.

With this design, users retain complete control over the input space and can produce a wide variety of outputs, while the adaptive mapping ensures that the outputs that most users would find distasteful are consistently avoided even as the underlying musical composition changes. This enables users to easily create rich, engaging and expressive music. Figure 1 shows a diagram of the adaptive interface.

In our study, we conjectured that participants might be discomfited by playing alone, so we employed a leadaccompaniment music paradigm where the user assumes the most prominent musical role and is supplemented by a backing track. Typically in group music creation the most prominent player would lead the rest, dictating the direction of the music. Here, the user's playing is still the most prominent aspect of the music, but the overall arc of the performance is pre-planned and the instrument facilitates traversing that arc. Within that track, the user still has substantial freedom and control, and thus can perceive a clear connection between the inputs they provide and the music they hear.

Our instrument is implemented in a web framework. For consistency in our research, our prototype only accepts keyboard input, but the design could also include mouse or touchscreen inputs. Delivering the instrument over the web is advantageous in two ways. First, from the user's perspective, the ubiquitiy of web technology ensures a low barrier to entry in terms of hardware and software prerequisites for playing the instrument. Second, from a research perspective, the webbased instrument can be tested on Mechanical Turk, improving our ability to collect data.

\section{Adaptivity}

The adaptivity of the instrument is its ability to change the mapping between inputs (key presses) and outputs (notes), as shown in Figure 1. In our experiment, all adaptation occurs in reference to the accompanying backing track. In other contexts the adaptation could take place without reference to a backing track, in which case the instrument would help guide the user through a solo performance.

For this prototype, a music expert provided a set of rules that 
dictated optimal note selections for the music in our experiments. Implementing a general technique for defining such rules represents an area of research in its own right, so we wished to remove that as a source of noise in the data. In the Future Work section we discuss some interesting possibilities for automatically inferring this mapping.

We implemented multiple levels of adaptivity, listed below in roughly increasing order of how much adaptation the user would be aware of while playing.

1. Static baseline case. For the baseline case, the instrument offers a static selection of pitches that are a run of adjacent notes from a chromatic scale (the scale that includes all notes on an acoustic piano). This is still a reduction from the infinite number of pitches that a computer could generate, but it is a reasonable baseline because Western music typically quantizes to this selection of pitches. In this baseline case, the instrument is non-adaptive.

2. Song-level adaptivity. The instrument offers a subset of the chromatic scale that best fits with the backing track as a whole. The selection does not change over the course of the performance, but would be different for a different backing track.

3. Section-level adaptivity. The backing track is partitioned into sections, and for each section the instrument offers a subset of the chromatic scale that best fits with that section. The method for finding sections can vary. For our experiment, we defined a section as a portion of the track that is oriented around the same tonal center. The selection changes a few times during the performance.

4. Chord-level adaptivity. The instrument follows the harmonic progression of the backing track, offering a subset of the chromatic scale that fits with each chord. The selection changes frequently during the performance.

There are many other ways that the interface could adapt, such as adding or removing input keys or dynamically adjusting the accompaniment. For our experiment we employed only the adaptive technique of changing the pitch selection at these three different levels of granularity (song-level, sectionlevel and chord-level). There could also be further gradations between these extremes, and more fine-grained adaptivity is possible than chord-level (e.g. beat-level). There are many possibilities for further investigation of adaptive techniques in this context, but for our experiment these adaptive modes are sufficient.

\section{EXPERIMENTAL SETUP}

Our investigation compared the efficacy of the different interface adaptation styles outlined above. In addition, we looked at the impact of the level of complexity of the overall musical performance. We also expected that differing levels of music experience would play a role. In accordance with our goal of enabling music creation without prior experience, we focus more on subjects who are not experts in music. Subjects' musical expertise was self-reported along with standard demographic information, although with Mechanical Turk the demographic information cannot be verified so we did not rely on it for our results. In addition to evaluating participants' creativity with the Guilford Alternative Uses Task [9], we collected subjective satisfaction ratings about the music creation experience. For these ratings, participants used a Likert scale to rate their satisfaction with the music they created. All music improvisation activities were approximately 80 seconds in duration.

The study consisted of the following stages:

1. Pre-questionnaire for demographic and music experience self-reporting.

2. Listening test (quality assurance, did not affect subsequent stages).

3. Music activity cognitive prime (independent variable, varied by condition).

4. Creativity assessment cognitive task (dependent variable).

5. Post-questionnaire for subjective metrics (dependent variable).

Subjects were recruited through the Mechanical Turk labor market. We restricted our subjects to English-speaking residents of the USA. To ensure our participants tried their best, we required that they have an excellent quality record with at least 95\% successful completions in Mechanical Turk.

The listening test, on which subjects listened to a short musical excerpt and checked the boxes for the instruments they heard, provided a further quality assurance measure. This test was not intended to eliminate any subjects, but rather just ensure that subjects were prepared for the music activity in terms of having their speakers or headphones ready, and were paying attention to what they heard. The rest of the study remained the same regardless of a participant's score on the listening test, but results from any subjects who failed to finish the test were rejected.

All results presented from the Mechanical Turk trials are from between-subject comparisons. We chose betweensubject over within-subject comparisons because we did not know how long the cognitive prime effect would last and were therefore concerned that in a withinsubject comparison we would not be able to isolate the priming effects. In total, we had the following experimental conditions (participant counts in parentheses) Unprimed baseline condition (50).

Control condition, subjects listened but did not play (25).

Played simple music with static instrument (25).

Played simple music with song-level adaptivity (25).

Played complex music with static instrument (25).

Played complex music with chord-level adaptivity (25).

Played complex music with section-level adaptivity (25).

Played complex music with song-level adaptivity (25).

\section{Creativity Metric}

Our quantitative creativity metric is based on Guilford's Alternative Uses Task, a standard psychological test of creativity on which subjects are asked to come up with alternative uses for a common household item [9]. We chose this task because it was used successfully by Lewis et. al to measure the 


\section{Overall Creativity Score}

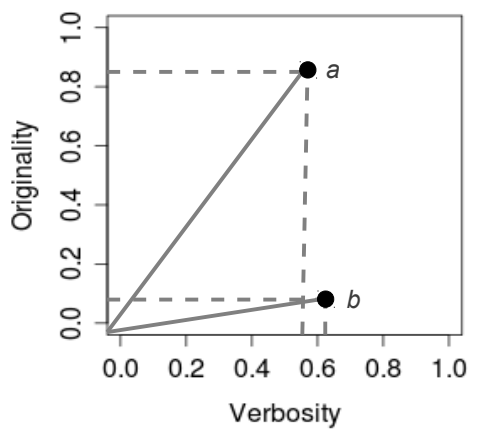

Figure 2. To create a single summary score for creativity, we compute the Euclidean norm of the verbosity and originality scores from Guilford's Alternative Uses Task. In this example, though both responses $a$ and $b$ have similar verbosity scores, the difference in the length of the solid lines shows that response $a$ earns a substantially higher overall score due to its higher originality.

impact of affective priming [14]. Also, as we are interested in providing a relative measurement between experimental conditions rather than a deep evaluation of individual subjects, the simplicity of Guilford's task is appealing for reproducibility and consistency.

In our study, the metric was administered as follows. First, subjects were presented with instructions informing them that they would be given one minute to come up for unusual uses for an object to be specified. Next, subjects clicked a button to begin, at which point a timer began counting down one minute and the subjects were shown the name of the object for which to list uses as well as a text box in which to enter their response. The prompt was one of "a brick," "a paperclip" or "an empty wine bottle." When the timer was up, the response was recorded and the study concluded. We note that one minute is a short time period in which to perform this task, and subjects might have provided more creative answers given more time. This prevents us from measuring the duration of any priming effects, but is sufficient to show significant differences in the responses.

The responses are graded according to the number and detail of uses generated as well as how unusual the uses are compared to responses from other participants [1]. All gibberish or nonsense responses are eliminated from the results. We measure the first criterion by looking at the length of the participant response, and call this score score verbosity. For the second criterion, we compute $\operatorname{Total}(x)$ as the total occurrences of a particular answer $x$ across all subjects, and then for an individual subject's response we weight each provided answer $x_{i}$ by $\frac{1}{\operatorname{Total}\left(x_{i}\right)}$ and sum the results. This gives higher values for unusual answers, and we call this score originality. These measurements can be made by simple counting, minimizing the possibility of subjective rater variance.

A typical application of creativity metrics like Guilford's Task would be to gain detailed insight into the mental faculties of an individual. In contrast, we need to make

\section{Musical Improvisation Priming Creativity}

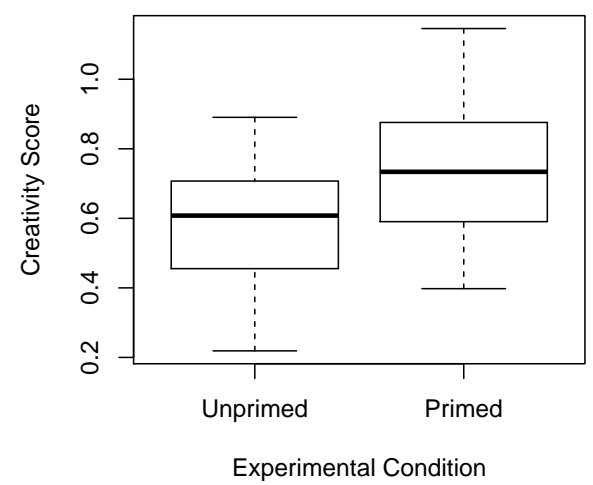

Figure 3. This boxplot shows the median and quartiles of creativity scores for subjects who were not primed versus subjects who were primed by participating in the most basic musical improvisation activity (25 subjects per condition). The creativity score is calculated using the method described in Figure 2. We see that participants in the primed condition achieve higher creativity scores $(p<.005)$, confirming our first hypothesis that musical improvisation can serve as a cognitive prime for creativity.

high-level comparisons between different experimental conditions. Thus, in our case it makes sense to flatten the multidimensional result down to a single overall creativity score. For an overall score, we normalize the two scores and compute the Euclidean norm, as shown in Figure 2. Though some information is lost in the dimensionality reduction, having a single dependent variable provides clearer results. Treating these dimensions independently warrants further investigation, but for this work we use the combined metric.

\section{RESULTS}

At a high level, our results confirm our hypotheses, but effects vary across some of the participant stratifications. Figure 3 shows the creativity scores for non-primed participants versus participants in our most basic music improvisation priming condition. The music improvisation activity consisted of improvising simple music using a non-adaptive interface on our web-based instrument. Participants who were primed demonstrate higher creativity than the unprimed participants, as measured using the overall creativity metric we defined in Figure 2. The difference is statistically significant $(p<.005)$. This shows that in general improvising music provides a cognitive boost to creativity, confirming our first hypothesis. In subsequent analyses, we will discuss the priming effects in terms of improvement over the non-primed condition, enabling us to make normalized comparisons between trials

This establishes a basic relationship between musical improvisation and measured creativity. For much of our analysis it is useful to consider music experts separately from the other subjects. We define music experts to be participants who described themselves as having "a lot" or "professional" music experience, and non-experts to be participants who described their music experience as "some," "a little" or "none." In general we find that results for this non-expert group are also 
Instrument Adaptivity in Simpler Music

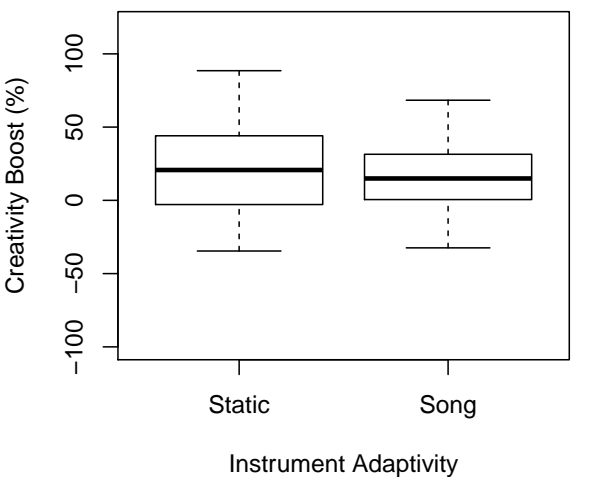

Figure 4. This boxplot shows the median and quartiles of creativity improvement over the non-primed baseline case for subjects primed by creating music using a static (non-adaptive) interface versus subjects primed by creating music using an interface with song-level adaptivity ( 25 subjects per condition). The participants in both these primed conditions showed improvement in creativity over the non-primed baseline, but we see that there is no statistically significant difference between the adaptivity modes. Thus, we conclude that adaptivity does not impact the creativity boost when playing simpler music.

applicable when further restricted to only participants who described their music experience as "a little" or "none."

In several cases, we see divergent results for the expert group compared to the rest, so it makes sense to consider this group separately. However, the limited number of experts in our sample prohibits detailed analysis. The expert group is also less relevant to our goal of supporting creative thinking in general, as most people are not music experts.

We cannot accurately verify demographic information on Mechanical Turk. Nevertheless, we ran ANOVA null-hypothesis tests on self-reported demographic variables, but did not find statistically significant effects. As most participants reported their age in the 20's or 30's, there might be effects due to age that could be uncovered in a larger sample. This merits further research, but is not the focus here.

\section{Instrument Adaptivity for Boosting Creativity}

Having established that the prime does provide a measurable creativity boost, we can break this down in more detail. On the simpler music trial, section- and chord-level adaptivity are indistinguishable from song-level adaptivity because the chords and sections are uniform. Thus, in this trial, the only adaptive modes available are no adaptivity or song-level adaptivity. We find that in this case adaptivity has no effect, as shown in Figure 4. Though both the nonadaptive case and song-level adaptivity primes provide statistically significant creativity boosts over the baseline unprimed case $(p<.05)$, the difference between them is not significant.

For more complex music, we found that different levels of adaptivity did result in differing levels of creativity boost for the non-expert participants, as shown in Figure 5. Only the conditions with song-level adaptivity and chord-level adap-
Instrument Adaptivity for Non-Experts Playing Complex Music

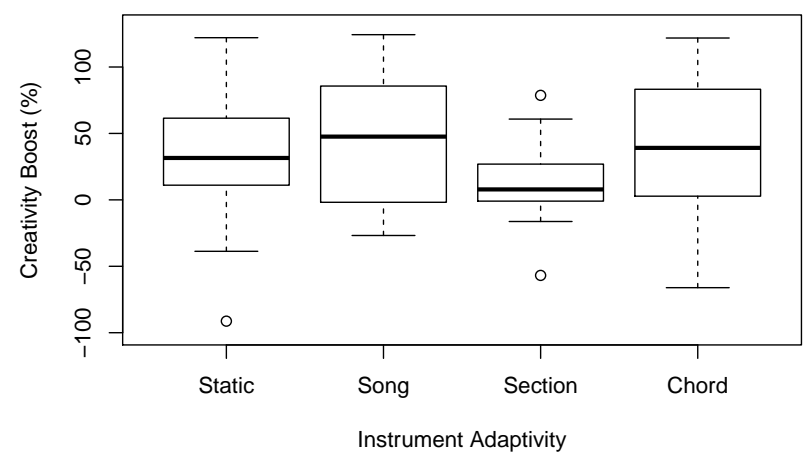

Figure 5. This boxplot shows the median and quartiles of creativity improvement over the non-primed baseline case for non-experts playing complex music on interfaces with different levels of adaptivity (20 subjects per condition). Improvising using the instruments with song- and chord-level adaptivity provided a statistically significant improvement over the non-primed baseline, but improvising using the static (nonadaptive) and section-level adaptive instruments did not. This suggests that struggling to play music (e.g. attempting complex music on the nonadaptive instrument) does not provide positive cognitive effects, but that this is mitigated by the adaptive instrument.

Music Complexity and Instrument Adaptivity for Non-Experts

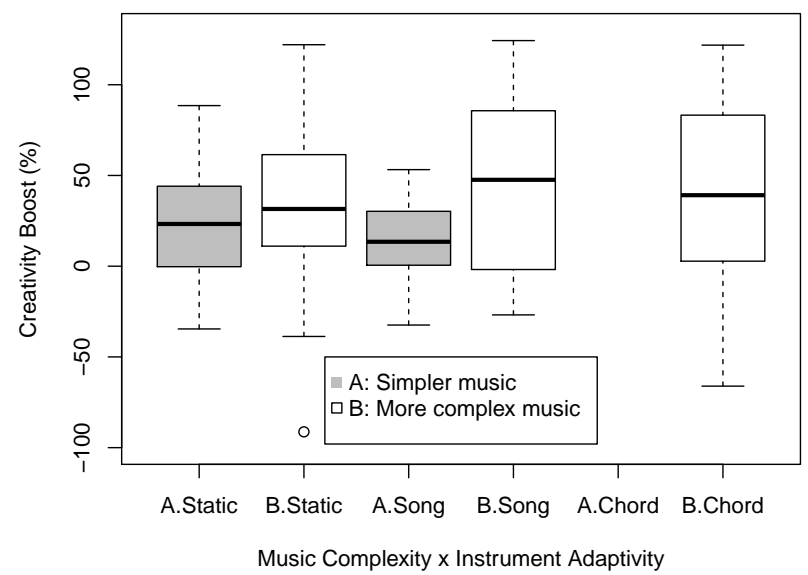

Figure 6. This boxplot shows the median and quartiles of creativity improvement over the non-primed baseline case for non-experts playing simple music (A, shaded) versus complex music (B, unshaded), across three different adaptivity modes ( 20 subjects per condition). For simple music, we omit chord-level because it is the same as song-level, and we omit section-level entirely because of our previous finding that it does not provide a cognitive benefit. Overall, playing more complex music on an adaptive instrument provides the most effective cognitive prime. 
tivity show statistically significant creativity boosts above the baseline $(p<.05)$; the non-adaptive and section-level adaptivity conditions do not show statistically significant improvement. This suggests that if the music improvisation is difficult then the activity is less effective as a cognitive prime, but that this is mitigated by the adaptive interface.

These results also indicate that section-level adaptivity is not as helpful as other adaptivity modes. To illuminate this further, we collected some qualitative feedback comparing the three adaptivity modes. Subjects reported that the infrequent sudden changes in section-level adaptivity were startling, in contrast with the single fixed adaptation in song-level adaptivity or the continual changing of chord-level adaptivity. We therefore conclude that section-level adaptivity is a disorienting "unhappy medium" for music improvisation.

We have so far considered adaptivity on both simpler and more complex music independently, and found that adaptivity does help non-experts playing complex music. We now look at the interaction of instrument adaptivity and music complexity for non-experts, shown in Figure 6. We find that, between simple versus complex music trials, there is not a statistically significant difference for the non-adaptive condition. However, improvising more complex music with songor chord-level adaptivity offers greater creativity boosts than improvising simpler music using either the adaptive or nonadaptive modes. An ANOVA indicates that the difference in music complexity is statistically significant $(p<.05)$. We note that these results do not hold for the expert case, and more data is necessary to illuminate the effect of adaptivity on creativity for music experts.

Taken together, these findings address our second hypothesis that instrument adaptivity magnifies the effect of improvising music as a cognitive prime for creativity. Our results show that, while adaptivity makes little difference for simple music, non-experts benefit from adaptivity when playing more complex music. Furthermore, playing complex music on an instrument with song-level or chord-level adaptivity offers greater benefit than playing simpler music. We generalize this by saying that the best musical cognitive prime for non-experts is improvising complex music on an instrument with song-level or chord-level adaptivity. This is a qualified confirmation of our second hypothesis.

\section{Instrument Adaptivity and Subjective Metrics}

To address our third hypothesis, that an adaptive instrument offers a more satisfying music creation experience, we look to the subjective satisfaction ratings. Here the trends are not as strong as with the creativity metric, but there are two notable observations. Figure 7 shows that for non-experts the overall trend of the satisfaction metric mirrors the boost measured with our creativity metric (presented earlier in Figure 5). Both song- and chord-level adaptivity show improvement, whereas section-level adaptivity and the non-adaptive interface are less strong. However, unlike with the creativity metric, the measured differences are not statistically significant. This suggests that even though the cognitive prime had a measurable impact, participants may not perceive effects directly.
Satisfaction for Non-Experts Playing Complex Music

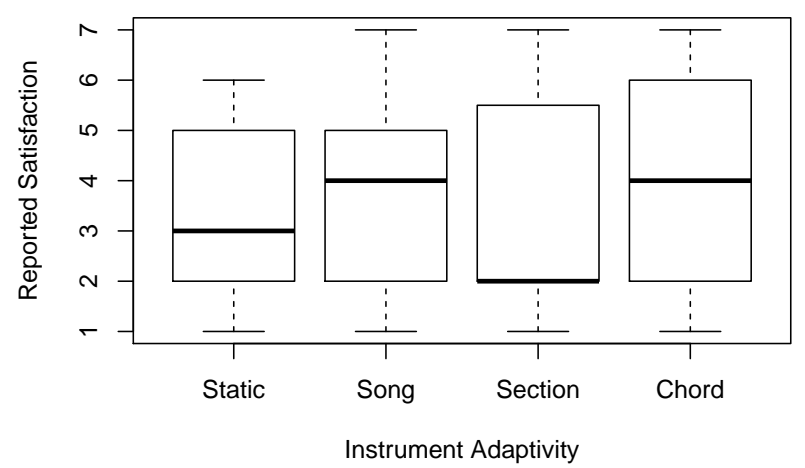

Figure 7. This boxplot shows the median and quartiles of the subjective satisfaction reported by non-experts playing complex music with different levels of adaptivity ( 20 subjects per condition). We see that the overall shape mimics the findings with the creativity metric (Figure 5), with song- and chord-level adaptivity cases outperforming non-adaptive (static) and section-level adaptivity cases. However, the differences in satisfaction ratings are not statistically significant.

Satisfaction for Experts Playing Complex Music

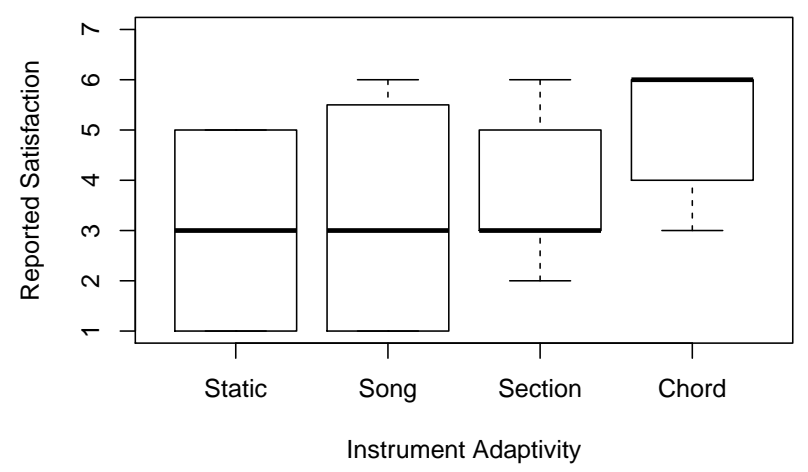

Figure 8. This boxplot shows the median and quartiles of the subjective satisfaction reported by experts playing complex music with different levels of adaptivity ( 5 subjects per condition). We see that increased adaptivity tends to result in monotonically greater satisfaction for music experts, which contrasts with the low satisfaction for section-level in overall ratings (Figure 7). Due to small sample size, the differences are not statistically significant, but if the axes are treated as numeric data the Pearson Correlation Coefficient is 0.42, suggesting a positive relationship between adaptivity and expert satisfaction. 
Listening Test Score Distribution

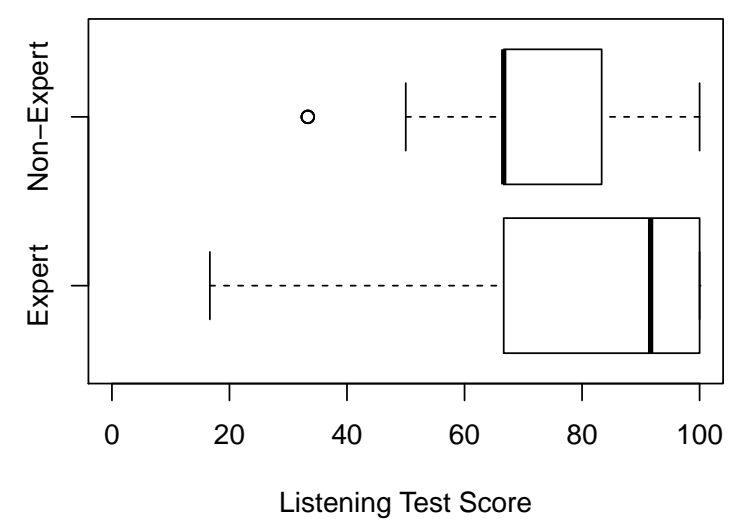

Figure 9. This boxplot shows the median and quartiles of scores on the listening test for experts versus non-experts ( 30 subjects per condition). It illustrates that, although music experts did outperform non-experts by median score, most subjects performed quite well on the listening test. There is not a statistically significant difference between the groups.

Figure 8 shows that music experts exhibit a different trend. For experts, the satisfaction ratings increases monotonically as the level of specificity of adaptation increases. This contrasts with our other results, in which section-level adaptation fares worse than other modes of adaptivity. The relationship can be quantified by treating least-to-most satisfaction and least-to-most specificity of interface adaption as numerical quantities, in which case they are correlated with a Pearson's Correlation Coefficient of .42. However, this makes assumptions about the nature of the data that may not be valid, and the sample size is such that we do not find statistically significant differences. Thus, we cannot draw firm conclusions, but the data suggests that adaptivity may be preferred by music experts.

Overall, this shows some support for the hypothesis that instrument adaptivity improves satisfaction, especially for music experts, but the results in this area are not definitive. This finding itself is interesting, however, as it means the priming measured by the creativity metric is not necessarily reflected in the subjective experience of the individual playing music.

\section{Good Listening Abilities Magnify Creativity Boost}

We included a music listening test in our trials that was intended to ensure participants were prepared before beginning the priming activity. However, as the listening test also provides a rough estimate of the quality of a subject's "musical ear," we look at how the listening test scores relate to our metrics. It is important to convey that the listening test was not especially challenging. The distribution of scores in Figure 9 shows that most participants received good scores, and we further note that approximately $25 \%$ of non-experts achieved a perfect score.

We find that this measure of listening ability is a strong predictor of the potency of the priming effects. For the non-
Adaptivity for Non-Experts With Good Listening Ability

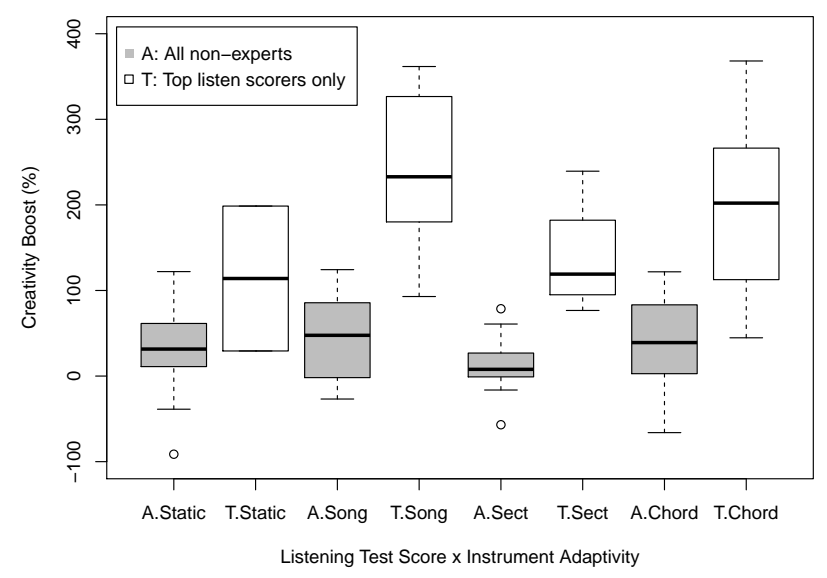

Figure 10. This boxplot shows the median and quartiles for creativity improvement over the non-primed baseline case for non-experts with good listening ability (T, unshaded, 5 subjects per condition) versus all non-experts (A, shaded, 20 subjects per condition) across four adaptivity modes. For the $\sim \mathbf{2 5 \%}$ of non-experts who received a perfect score on the listening test ( $T$, unshaded), the creativity score improvements are quadruple the creativity score improvement for non-experts overall (A, shaded) in every adaptivity category. The difference is statistically significant $(p<.001)$. This shows that playing music is a very effective cognitive prime for creativity in people with good listening abilities, even if they haven't had musical training. This also demonstrates that using an adaptive interface for music improvisation can magnify the cognitive benefits.

experts who earned perfect scores on the listening test, the creativity score improvement increases by approximately a factor of four. This is shown in Figure 10. The difference is especially dramatic for the song-level and chord-level adaptivity conditions.

The effect is further magnified when excluding all but the lowest two levels of reported music experience ("none" or "a little"). The best improvement is for participants with "none" or "a little" music experience who were primed by improvising more complex music on an instrument with song-level adaptivity. The median creativity score improvement in this case is $400 \%$ (i.e., the median score for this condition is five times the median score of the baseline non-primed condition). These observed differences are statistically significant. Relaxing the listening test cutoff to include the top $\sim 50 \%$ scorers among non-experts reduces but does not eliminate the increase compared to the overall non-expert group.

These effects far outpace the differences between the expert and non-expert groups. This shows that the instrument adaptivity is well-suited to people who have an ear for music, even if they haven't had any musical training. Similarly, we can conclude that while improvising music on the instrument primes creative thinking across the board, the benefit is magnified for individuals with an ear for music.

\section{Qualitative Results}

There are a few qualitative reports that supplement the quantitative findings described above. Feedback subjects provided 


\section{Adaptive Instrument Note Play Counts}

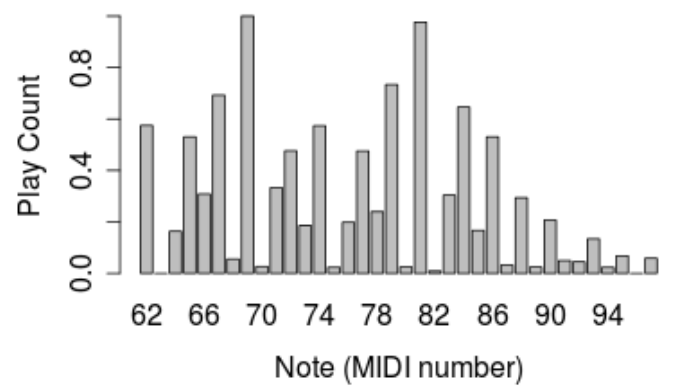

Figure 11. This histogram shows how often the subjects played different notes on the chord-level adaptive instrument. Although the data is interesting, showing patterns beyond what can be explained by the adaptivity choices alone, an analysis of the musical content is not the focus of this paper. We include the graph to show that our system can collect rich quantitative data about the musical performances of the subjects on Mechanical Turk.

during the small-scale in-person trials helps illustrate what works well on the adaptive instrument. For the in-person trials, subjects tried playing the instrument under all the different adaptivity conditions, and then were asked to compare their experiences. Subjects reported that "the [non-adaptive] one didn't sound as good," whereas "the [song-level adaptation] chose the best sound to fit." One subject reported feeling confused when the instrument changed the mapping. Another subject, when asked if any version had felt limiting, immediately identified the non-adaptive version. This is interesting because the non-adaptive instrument in a real sense offers greater flexibility in terms of the variety of pitches that are available, but it is experienced as limiting when compared to the adaptive technology.

\section{Implications for Quantitative Music Analysis}

In addition to evaluating the cognitive impact of the musical priming task, we collected data about the musical task itself in the form of note onsets. By aggregating this data, we can expose the implicit musical preferences expressed by the participants during the musical improvisation, as shown in Figure 11. There are clearly some notes that are consistently preferred, which indicates that the data is worth examining, but as the musical content is not the focus of this paper, we do not include an analysis here. Nevertheless, this demonstrates another strength of our approach, which is that we can collect note-by-note statistics for both amateur and expert musical improvisation with significant numbers of participants. We will use this data to examine implicit musical preferences in order to further our understanding of music cognition and improve future interface designs.

\section{FUTURE WORK}

There are a number of opportunities to extend this research. Our findings surrounding expert musicians were hampered by small sample size, so more in-depth investigation is necessary in that area. In addition, the precise effects of the cognitive prime warrant more careful delineation. This includes comparing the different axes of creativity measured on Guilford's
Alternative Uses Task, as interesting trends may have been blurred due to dimensionality reduction.

One limitation of this study is that we did not pin down the psychological mechanism underlying the priming we observed. The music may have functioned as an affective prime that improved the mood or motivation of the subjects. Alternatively, the benefits may stem from the improvisation having moved participants into a mental state of spontaneous idea generation, which subsequently facilitates the verbal idea generation measured on the task. Both the motivation explanation and the idea of "warming up" for idea generation are supported by the literature on creative cognition $[3,28]$. More sophisticated psychological measurements could illuminate how this priming occurs.

There are several ways in which the adaptive instrument can be improved and enhanced. A natural extension is to elminate the need for rule-based adaptation in favor of a more automated approach. We chose to rely on a tailored design from a domain expert in order to reduce noise in our measurements, but existing research in the area of music information retrieval supports the feasibility of a fully automated system that analyzes the track and selects output pitches based on music theory heuristics. This would open the door to far wider uses of the music creation technology by enabling improvisation with arbitrary music tracks.

Another promising avenue for the instrument is in collaborative composition. In this paper, the instrument adapted to a fixed backing track. However, the same adaptive techniques could adapt multiple instances to each other at the same time. This would facilitate multiple people creating music together, which could have broad implications for supporting positive group dynamics and brainstorming sessions.

Finally, a valuable aspect of this work is its real-world application in the context of creativity support tools. We intend to explore the possibilities for integrating music improvisation as a priming activity within the framework of existing tools. Our method can augment current strategies for supporting creative work, and investigating the best method for integrating our techniques is an important future direction.

\section{CONCLUSION}

Creativity is emerging as one of the most valuable cognitive skills, and the field of HCI is uniquely poised to develop supporting technologies in this area. In 2009, Shneiderman proposed creativity support tools as a grand challenge for the field of HCI [22]. Our work shows that music improvisation as a cognitive prime is a fruitful avenue to explore, and we demonstrate the power of an adaptive interface in the realm of creative expression. This represents one novel approach in the greater context of creativity support tools, but it is only the beginning of the tremendous opportunity for the HCI community as a whole to address this important and complex domain.

\section{ACKNOWLEDGEMENTS}

The authors gratefully acknowledge support from the NSF (IIS-1065154). 


\section{REFERENCES}

1. Amabile, T. Social psychology of creativity: A consensual assessment technique. Journal of Personality and Social Psychology 43, 5 (1982), 997-1013.

2. Amabile, T. The social psychology of creativity: A componential conceptualization. Journal of Personality and Social Psychology, 45 (1983), 357-376.

3. Amabile, T. Motivation and creativity: Effects of motivational orientation on creative writers. Journal of Personality and Social Psychology 48, 2 (1985), 393.

4. Amabile, T., Barsade, S., Mueller, J., and Staw, B. Affect and creativity at work. Administrative Science Quarterly 50, 3 (2005), 367.

5. Camurri, A., and Ferrentino, P. Interactive environments for music and multimedia. Multimedia systems 7, 1 (1999), 32-47.

6. Črnčec, R., Wilson, S., and Prior, M. The cognitive and academic benefits of music to children: Facts and fiction. Educational Psychology 26, 4 (2006), 579-594.

7. Demorest, S., and Morrison, S. Does music make you smarter? Music Educators Journal 87, 2 (2000), 33-58.

8. Fredrickson, B. What good are positive emotions? Review of general psychology 2, 3 (1998), 300.

9. Guilford, J. The Nature of Human Intelligence. McGraw-Hill, New York, NY, USA, 1967.

10. Gurevich, M., and Treviño, J. Expression and its discontents: toward an ecology of musical creation. In Proceedings of the 7th international conference on New interfaces for musical expression, ACM (2007), 106-111.

11. Hewett, T., Czerwinski, M., Terry, M., Nunamaker, J., Candy, L., Kules, B., and Sylvan, E. Creativity support tool evaluation methods and metrics. Creativity Support Tools (2005), 10-24.

12. Ipeirotis, P., Provost, F., and Wang, J. Quality management on amazon mechanical turk. In $A C M$ SIGKDD Workshop on Human Computation, ACM (2010), 64-67.

13. Kittur, A., Chi, E., and Suh, B. Crowdsourcing user studies with mechanical turk. In CHI 2008, ACM (2008), 453-456.

14. Lewis, S., Dontcheva, M., and Gerber, E. Affective computational priming and creativity. In Proceedings of the 2011 Annual Conference on Human factors in Computing Systems, CHI '11, ACM (New York, NY, USA, 2011), 735-744.

15. Lombardi, W., Higgins, E., and Bargh, J. The role of consciousness in priming effects on categorization assimilation versus contrast as a function of awareness of the priming task. Personality and Social Psychology Bulletin 13, 3 (1987), 411-429.

16. Machover, T., and Chung, J. Hyperinstruments: Musically intelligent and interactive performance and creativity systems. In Proceedings of the International
Computer Music Conference (Columbus, OH, USA, 1989).

17. Mammarella, N., Fairfield, B., and Cornoldi, C. Does music enhance cognitive performance in healthy older adults? the vivaldi effect. Aging clinical and experimental research 19, 5 (2007), 394.

18. Momeni, A., and Wessel, D. Characterizing and controlling musical material intuitively with geometric models. In Proceedings of the 2003 conference on New interfaces for musical expression, National University of Singapore (2003), 54-62.

19. Mulder, A. Virtual musical instruments: Accessing the sound synthesis universe as a performer. In Proceedings of the First Brazilian Symposium on Computer Music (1994), 243-250.

20. Ross, J., Irani, L., Silberman, M., Zaldivar, A., and Tomlinson, B. Who are the crowdworkers?: Shifting demographics in mechanical turk. In Extended Abstracts CHI 2010, ACM (2010), 2863-2872.

21. Schellenberg, E. Music and cognitive abilities. Current Directions in Psychological Science 14, 6 (2005), 317-320.

22. Shneiderman, B. Creativity support tools: A grand challenge for hci researchers. Engineering the User Interface (2009), 1-9.

23. Shneiderman, B., Fischer, G., Czerwinski, M., Resnick, M., Myers, B., Candy, L., Edmonds, E., Eisenberg, M., Giaccardi, E., Hewett, T., Jennings, P., Kules, B., Nakakoji, K., Nunamaker, J., Pausch, R., Selker, T., Sylvan, E., and Terry, M. Creativity support tools: Report from a u.s. national science foundation sponsored workshop. International Journal of Human-Computer Interaction 20, 2 (2006), 61-77.

24. Simonton, D. Origins of genius: Darwinian perspectives on creativity. Oxford University Press, New York, NY, USA, 1999.

25. Sternberg, R. Handbook of creativity. Cambridge University Press, 1998.

26. Thompson, W. F. Music, thought, and feeling: understanding the psychology of music. Oxford University Press, New York, NY, USA, 2009.

27. Tomasco, S. Ibm 2010 global ceo study: Creativity selected as most crucial factor for future success. IBM Press Release, http://www-03.ibm.com/press/us/en/ pressrelease/31670.wss, 2010.

28. Torrance, E. The nature of creativity as manifest in its testing. The nature of creativity (1988), 43-75.

29. Wessel, D. Instruments that learn, refined controllers, and source model loudspeakers. Computer Music Journal (1991), 82-86.

30. Wessel, D., and Wright, M. Problems and prospects for intimate musical control of computers. Comput. Music J. 26, 3 (Sept. 2002), 11-22. 